\section{"Jugoslawen" in der Schweiz}

Soziale, kulturelle und ethnische Herkunft, Integrationsprobleme

N. Boškovska

Die ersten jugoslawischen Fremdarbeiter kamen in den sechziger Jahren in die Schweiz. Ihre Zahl blieb aber zunächst gering. 1970 waren 54\% aller Ausländer Italiener, 11,2\% Spanier, 11\% Deutsche, 5,2\% Franzosen, 4,1\% Österreicher und nur 2,3\% Jugoslawen (knapp 25000 Personen). Diese gehörten vorwiegend "höheren Berufskategorien" an. Rund die Hälfte waren Akademiker oder andere Angehörige intellektueller Berufe. In den Spitälern arbeiteten neben Ärzten zahlreiche Krankenschwestern, Pflegerinnen und Pfleger. Ungelernte Arbeitskräfte aus Jugoslawien fanden damals in der Schweiz kaum Beschäftigung, da diese Posten bereits durch Italiener besetzt waren. Die Arbeitgeber, so konnte man in der Zeitung lesen, waren zufrieden mit den Leistungen und dem Verhalten der Jugoslawen. ${ }^{1}$

Diese ersten Fremdarbeiter aus Jugoslawien stammten vor allem aus den nördlichen, stärker entwickelten Landesteilen, insbesondere aus Kroatien. Die Schweizer Volkszählungen unterschieden die Jugoslawen zwar nicht nach nationaler Herkunft. Aus den Angaben über Religionszugehörigkeit und Muttersprache kann man dennoch bis zu einem gewissen Grad Rückschlüsse auf die Nationalität ziehen.

1970 waren von den 24971 Personen mit jugoslawischer Staatsbürgerschaft 14143 römisch-katholisch (56\%), also mit grösster Wahrscheinlichkeit Kroaten und Slowenen. Sie kamen aus einer Gegend, deren Kultur mitteleuropäisch geprägt ist.

6897 Personen waren orthodoxe Christen, also Serben, Makedonier, Montenegriner und damit Menschen, deren Heimat jahrhundertelang unter osmanischer Herrschaft gestanden hatte. 1094 Personen waren Muslime, die meisten wohl Slawen, das sieht man an der Kategorie "Hauptsprache». Nur 10,7\% waren Kinder unter 15 Jahren. $^{2}$

\footnotetext{
1 TA, 19.7.1969.

2 Quelle: Bundesamt für Statistik.

3 Quelle: Bundesamt für Statistik.
}

Im Laufe der siebziger Jahre stieg die Zahl der jugoslawischen Bevölkerung in der Schweiz auf 61000 , was aber immer noch wenig ins Gewicht fiel. Die Angaben der Volkszählung von 1980 lassen aber bereits eine allmähliche Veränderung der Zusammensetzung erkennen. Die Katholiken bildeten mit 20719 Personen zwar immer noch die grösste Gruppe, aber nur noch knapp. Die Orthodoxen waren nun fast gleichauf (18 707). Die Zahl der Muslime hatte sich beinahe verzehnfacht auf 10623 Personen, von denen knapp 4000 vermutlich Albaner waren. Der Anteil der Kinder war auf 15,6\% gestiegen. ${ }^{3}$

Die Integration dieser ersten Fremdarbeitergeneration verlief insgesamt reibungslos. Der wachsende Schweizer Arbeitsmarkt absorbierte die häufig gut qualifizierten und motivierten Kräfte problemlos. Sie selbst wollten in erster Linie arbeiten, Geld verdienen und sparen und irgendwann zurückkehren. Die kleine Zahl erlaubte es ihnen nicht, Ghettos zu bilden und sich als Gruppe abzukapseln. So lernten die meisten, anders als die Italiener, schnell Schweizerdeutsch. Für die Kinder wünschten sie sich eine möglichst hochstehende Ausbildung. Bildungsbewusstsein war ein herausragender Zug dieser Emigrantengeneration. Jugoslawische Eltern waren unglücklich, wenn ihr Kind sich mit einer Lehre begnügen musste.

Noch Mitte der achtziger Jahre galten etwa für die deutschen Behörden die Jugoslawen als vollintegrierbare und problemlose "Traumausländer". Sie versuchten nicht aufzufallen und grenzten sich etwa von den Türken ab, die - so eine junge Jugoslawin - «mit ihrem grossen schwarzen Schnauzbart herumlaufen und mit Frau und einer Horde Kinder im Schlepptau provozierend auf der Strasse gehen. Man darf nie vergessen, dass man hier Ausländer ist, und muss sich möglichst ruhig verhalten."

Diese Einstellung, dass man sich als Ausländer so gut wie möglich anzupassen habe und nicht negativ auffallen sollte, ist für kleine Gemeinschaften bezeichnend. Je grösser und vor allem auch besser organisiert eine Gruppe ist, um so selbstbewusster tritt sie auf und fordert ihrerseits Anerkennung und Tolerierung ihrer Eigenheiten.

Die achtziger Jahre brachten den quantitativen und qualitativen Sprung bei der jugoslawischen Population in der Schweiz. Die Volkszählung von 1990 zeigt das deutlich. Zum einen ist die Zahl der Jugoslawen auf 172777 gestiegen und hat sich somit innerhalb von zehn Jahren fast verdreifacht. Auch die Zusammensetzung ist eine gänzlich andere geworden: Das Verhältnis zwischen Männern und Frauen ist nun sehr einseitig geworden: Auf eine Frau kommen 1,54 Männer, 1970 waren es noch 1,16 gewesen.

Die Katholiken, also jene Gruppe, welche der schweizerischen Kultur und Lebensweise am nächsten steht, ist anteilmässig von einem Drittel 1980 auf weniger als einen Viertel geschrumpft (40 000 Personen, 22,7\% gegenüber 34\% 1980). Die orthodoxen Christen sind nun in grösserer Zahl da (46 $511=27 \%$ ). Mit 55453 oder 32\% bilden neu die Muslime deutlich die stärkste Gruppe. Zehn Jahre zuvor, 1980,

hatte ihr Anteil noch 17,4\% betragen. Jene, die unter

Korrespondenz:

Dr. phil. Nada Boškovska

Höfliweg 7

CH-8055 Zürich

E-mail: bonada@hist.unizh.ch 
die Sprachgruppe «übrige» fallen und in erster Linie als albanischsprachig identifiziert werden können, machen neu 21\% aus gegenüber 6\% im Jahr 1980. 1970 hatten sie noch gar keine statistische Relevanz. Gleichzeitig bilden sie den grossen Teil der muslimischen Gruppe.

Aus sozialer Sicht ist von Bedeutung, dass 54\% der Personen, die 1990 einer bezahlten Arbeit nachgingen, ungelernte Arbeiter und Angestellte waren, während, wie wir gesehen haben, in den sechziger und siebziger Jahren vor allem qualifizierte Arbeitskräfte einwanderten. Und der Anteil der Kinder betrug mittlerweile $22,7 \%{ }^{4}$

Die Statistik widerspiegelt sehr klar die Charakteristika der Einwanderungsgeneration der achtziger Jahre. Die sich nach Titos Tod (1980) stetig verschlechternde wirtschaftliche Lage in Jugoslawien und die 1981 einsetzenden Spannungen im Kosovo bewogen immer mehr Menschen, v.a. aus den ärmsten Landesteilen, im Ausland Arbeit zu suchen. In die Schweiz kamen nun in erster Linie ungelernte Arbeitskräfte aus ländlichen Gebieten und somit Menschen, die bei der Integration in eine urbane, postmoderne Lebenswelt grössere Hürden überwinden müssen.

In den achtziger Jahren setzte somit eine Umstrukturierung der jugoslawischen Bevölkerungsgruppe in der Schweiz ein - eine Entwicklung, deren Tempo sich dann seit dem Ende der achtziger Jahre rasant beschleunigt hat. Und das aus folgenden Gründen:

Zwischen 1988 und 1990 wurde im Kosovo die vorher stark ausgebaute Autonomie aufgehoben und ein repressives Regime eingeführt. Zahllose Albanerinnen und Albaner wurden zudem aus dem Staatsdienst entlassen. Dies hatte zur Folge, dass viele Albaner aus politischen oder ökonomischen Gründen in die Schweiz kamen und hier entweder legal oder illegal arbeiteten oder ein Asylgesuch einreichten. Von 1989 bis 1991 gingen 22115 Asylgesuche von Personen aus Jugoslawien ein. Die allermeisten Bewerber stammten aus dem Kosovo. Und mehr als die Hälfte der 40000 aus Jugoslawien stammenden Saisonniers waren 1991 Kosovo-Albaner. ${ }^{5}$

Mit dem Beginn des Krieges in Jugoslawien im Jahre 1991 hat sich der Zustrom von - auch in der Statistik so genannten - Ex-Jugoslawen noch weiter verstärkt, insbesondere auch durch den Familiennachzug, so dass inzwischen diese Bevölkerungsgruppe an Zahlenstärke beinahe mit den Italienern gleichgezogen hat. 1997 lebten 316607 «Ex-Jugoslawen" gegenüber 344591 Italienern in der Schweiz.

Im weiteren soll hier insbesondere von der grössten Gruppe aus dem ehemaligen Jugoslawien die Rede sein, von den Albanern. Mit einer Zahlenstärke von mittlerweile gut 200000 Personen, zu denen noch einige Tausend Albaner aus Albanien hinzukommen, machen sie zwei Drittel der "ex-jugoslawischen" Bevölkerung in der Schweiz aus und prägen das Bild von den Jugoslawen stark. Dies ist insofern verzerrend, als die Albaner bei der letzten gesamtjugosla- wischen Volkszählung 1991 nur 9,3 \% der ganzen Bevölkerung ausmachten. Auch in bezug auf die Republik Makedonien ist das Verhältnis hier auf den Kopf gestellt: Während die Albaner in Makedonien einen Viertel der Bevölkerung bilden, leben in der Schweiz über 40000 in der Regel muslimische makedonische Albaner gegenüber vielleicht 8000 slawischen, christlichen Makedoniern. Der Makedonier in der Schweiz ist damit in der Regel ein Albaner.

Die Zahl von 200000 Albanern aus Kosovo und Makedonien (davon ca. 50000 Asylbewerber) heisst, dass fast $10 \%$ der Bevölkerung dieser beiden Gebiete hierher gekommen sind. Man findet dort kaum eine Familie ohne Verbindung zur Schweiz. ${ }^{6}$ Charakteristisch ist weiter, dass sich die Albaner hier gemäss ihren Herkunftsorten gruppieren. ${ }^{7}$ Dies alles bedeutet, dass wir in der Schweiz gewissermassen eine Abbildung der albanischen Gesellschaft haben. Das hat einerseits zur Folge, dass die Beziehungen zur Schweiz intensiv sind. Andererseits bringen aber die albanischen Immigranten ihre eigene Gesellschaftsform und ihre menschliche Umgebung mit, wenn auch, was nicht unwesentlich ist, in reduzierter Form. Aber dennoch sind sie weitgehend nicht darauf angewiesen, sich mit ihrer Schweizer Umwelt zu befassen. Sie bleiben eine sehr auf sich selbst bezogene Bevölkerungsgruppe, die sich für die Schweiz nicht interessiert. ${ }^{8}$

In bezug auf Lebensweise und Wertvorstellungen unterscheiden sich die fast durchwegs muslimischen und streng patriarchalischen Albaner weit stärker von der Schweizer Bevölkerung als die übrigen «Ex-Jugoslawen", die in der Regel slawisch und christlich sind. Dabei ist die Religion aber eher von sekundärer Bedeutung. Wichtiger ist, dass sich in den albanisch besiedelten, vorwiegend ruralen Gebieten eine Lebensform erhalten hat, in welcher die Grossfamilie und übergreifende Familienverbände, Clans, das massgebliche Bezugssystem sind. Die albanische Bevölkerung war daher in osmanischer Zeit genauso wie in jugoslawischer antistaatlich eingestellt. Ihr sozialer Rahmen war und ist die Familie, das Dorf, der Clan. Dort spielt sich das Leben nach gewohnheitsrechtlichen Gesetzen ab. Es gibt klare Regeln dafür, was in bestimmten Situationen zu tun ist. Der grösste Greuel war und ist den Albanern die Einmischung von aussen. ${ }^{9}$ Chancen, welche der Staat bot, wurden aber durchaus genutzt. So waren die Albaner im Osmanischen Reich in Militär und Verwaltung überproportional vertreten.

Alle die genannten Eigenschaften, v.a. die Familien- und Clanstrukturen, in denen das Gesetz des

\footnotetext{
4 Quelle: Bundesamt für Statistik.

5 Weltwoche, 21.3.1991.

6 Leuenberger U, Maillard A. Les damnés du troisième cercle. Les Kosovars en Suisse 1965-1999. Genève 1999, S. 21.

7 Leuenberger/Maillard, Les damnés, S. 22-23.

8 Leuenberger/Maillard, Les damnés, S. 28.

9 Roux M. Les Albanais en Yougoslavie. Minorité nationale, territoire et développement. Paris 1992, S. 245.
} 
Schweigens gegen aussen ohnehin gilt, sind eine gute Grundlage für die Entwicklung mafiöser Strukturen. Deshalb verwundert es nicht, dass die Albaner die Tamilen und Türken aus dem Heroingeschäft gedrängt ${ }^{10}$ und damit aber auch deren schlechten Ruf übernommen haben.

Die traditionelle Lebensweise und die überragende Bedeutung der Familie kommen u. a. im grossen Kinderreichtum zum Ausdruck. Nirgends in Europa ist die Geburtenrate auch nur annähernd so hoch wie im Kosovo: Im Durchschnitt bringt dort jede Frau zwischen 15 und 49 Jahren 6,6 Kinder zur Welt. Die Geburtenrate war in den sechziger Jahren sogar höher als Ende der dreissiger Jahre. Diese enorme Natalität hat im Kosovo zu einer Bevölkerungsexplosion geführt: Bei der Gründung des Staates Jugoslawien im Jahr 1918 war die Provinz etwas weniger dicht besiedelt als der jugoslawische Durchschnitt. In den neunziger Jahren lebten dort 180 Einwohner $/ \mathrm{km}^{2}$ gegenüber $92 \mathrm{im}$ gesamten Jugoslawien. ${ }^{11}$ Da die wirtschaftliche Entwicklung in keiner Weise mit diesem Wachstum Schritt hielt, war die Emigration die logische Folge.

Von überragender Bedeutung in der albanischen Gesellschaft ist das Konzept der Ehre eines Mannes, die - wie in extrem patriarchalischen Kulturen üblich zu einem guten Teil davon abhängt, ob er die Sexualität der Frauen seiner Familie unter Kontrolle halten kann und bei Verfehlungen gemäss Tradition und Erwartung eingreift. Das kann so weit gehen, dass er die eigene Schwester oder Tochter töten muss. Die Bereitschaft, die eigene Ehre - in welcher Situation auch immer - selbst mit Waffengewalt zu verteidigen, führt in einer nichtalbanischen Umwelt unweigerlich $\mathrm{zu}$ Spannungen, Konflikten mit dem Gesetz und gegenseitiger Verachtung. Die Albaner sind in den Augen der anderen gewalttätig und unzivilisiert; den Albanern erscheinen die einheimischen Männer als Feiglinge.

Für Mediziner ist die Tatsache von Interesse, dass traditionell lebende und das Kollektive betonende Gesellschaften in der Regel keine Formen der Auseinandersetzung und Beschäftigung mit sich selbst kennen, wie sie sich im Westen entwickelt haben. Die Menschen orientieren sich vielmehr an tradierten Werten und vorgegebenen Verhaltensweisen. Wenn sie dann in eine Umgebung kommen, die diese Werte mit ihnen nicht teilt und die Einhaltung ihrer Verhaltensnormen nicht kontrolliert, geraten sie in Orientierungsschwierigkeiten. Sie sind dann auf sich gestellt, sollten für ihre Probleme individuelle Lösungen finden. Sie haben jedoch nicht gelernt, sich mit sich selbst als Person zu befassen, vielmehr sind sie in eine Rolle hineingewachsen - in die Rolle der Ehefrau, Mutter, des Ehemannes, ältesten Bruders, des Familienoberhauptes usw.

Aber auch die Menschen aus den eher urbanen Gebieten des ehemaligen Jugoslawien waren in sozialistischer Zeit gewohnt, für viele Probleme eine Lösung von oben zu erwarten, von der Partei, vom Staat.
Bezogen auf die Gesundheit bedeutet das, dass dem Gesundheitssystem ebenfalls eine Erwartungshaltung entgegengebracht wird: Die Ärzte sollen mich mit ihrem Instrumentarium gesund machen, ein eigener Beitrag ist nicht vorgesehen. Die Patienten sind es vom Gesundheitswesen in der Heimat gewohnt, dass möglichst rasch mit möglichst starken Mitteln eingegriffen wird.

Was viele Menschen in Jugoslawien und im übrigen Osteuropa ausser Eigenverantwortung ebenfalls wenig entwickelt haben, ist eine staatsbürgerliche Einstellung. Es galt vielmehr, dem Staat möglichst wenig zu geben und möglichst viel von ihm zu nehmen. Es wird wohl eine Weile dauern, bis sich in den Nachfolgestaaten diese Mentalität ändert.

Der Krieg in Jugoslawien hat das schlechte Bild der Jugoslawen nicht etwa produziert, sondern nur bestätigt und verschärft. Bereits zu Beginn der neunziger Jahre war der Ruf der Jugoslawen gründlich ruiniert, das Schimpfwort "Jugo" etabliert. 1992 war die Abneigung gegen die Jugoslawen so gross, dass die sonst grosszügigen Schweizerinnen und Schweizer kaum bereit waren, für die Kriegsopfer zu spenden. Die Glückskette verzichtete deshalb darauf, einen eigentlichen Sammeltag durchzuführen.

Zunächst war es die Entwicklung der Kriminalität, die am meisten zum Hass gegen die Jugoslawen beitrug. Die ersten Fremdarbeiter, gleich welcher Herkunft, begingen weniger Straftaten als die einheimische Bevölkerung. Sie waren gekommen, um zu arbeiten und $\mathrm{zu}$ verdienen und hatten kein Interesse daran, ihre Aufenthaltsbewilligung aufs Spiel zu setzen. Zudem war der Staat damals weit weniger zimperlich und griff bei abweichendem Verhalten hart durch, nicht nur gegen Erwachsene, sondern selbst gegen Kinder. Auch die albanischen Fremdarbeiter der siebziger Jahre fielen nicht weiter auf. Sie waren fleissig und diskret und duldeten Normabweichungen ihrer Landsleute nicht. Wer sich nicht konform verhielt, wurde aus der Gemeinschaft ausgeschlossen. Allerdings integrierten sich die Albaner auch nicht, sondern praktizierten eine gewollte Isolation gegenüber der schweizerischen Bevölkerung. ${ }^{12}$ Und sehr selten liessen sie ihre Familie nachkommen.

Seit Mitte der achtziger Jahre nahmen in der Schweiz die schweren Formen der Kriminalität - Einbrüche und physische Gewalt - stark zu. Gleichzeitig wurde der Prozentsatz der ausländischen Straftäter immer grösser. Einer der Gründe: Der Anteil der Schweizer an der besonders involvierten Gruppe der 18- bis 40jährigen Männer ist rückläufig, während er bei den Ausländern stetig steigt. Zudem waren die Immigranten von den Krisenerscheinungen wie Arbeitslosigkeit und Lehrstellenmangel besonders betroffen. Und hier sind an erster Stelle die schlecht ausgebildeten und wenig integrationswilligen und -fähigen Jugoslawen der neuen Fremdarbeitergeneration,

10 Leuenberger/Maillard, Les damnés, S. 69.

11 Roux, Les Albanais, S. 144-146, 149.

12 Leuenberger/Maillard, Les damnés, S. 59-60. 
in erster Linie ethnische Albaner, zu nennen, die auch ein anderes Verhältnis zur Gewaltanwendung an den Tag legten. Sie hätten "zugegebenermassen die grössere Gewaltbereitschaft als jede Ausländergruppe zuvor", meint ein Berner Ethnologieprofessor. ${ }^{13}$

Ein weiterer Grund, warum gerade so viele junge Albaner in vielerlei Hinsicht auffällig und überdurchschnittlich straffällig werden und wurden, ist in ihrer Trennung von der Grossfamilie zu suchen. $\mathrm{Zu}$ Hause unterstehen die jungen Leute der strengen Kontrolle durch männliche Autoritäten. Wenn sie in die Schweiz kommen, fallen sie einerseits aus den Kontrollinstanzen heraus, andererseits müssen sie nun selbst Entscheidungen treffen, für die zu Hause andere zuständig waren und auf die sie somit nicht vorbereitet sind.

Der Krieg liess den Zustrom von Asylbewerbern, die ebenfalls meistens aus dem Kosovo stammen, anschwellen. Somit wuchs auch das Potential an der strafrechtlich überall besonders auffälligen Gruppe der jungen Männer, die sich in diesem Fall ausserdem in einer schwierigen Lebenssituation befinden. Verschärfend kommt noch hinzu, dass sich die Asylbewerber erklärtermassen nicht integrieren sollen, damit die Rückkehrbereitschaft erhalten bleibt. ${ }^{14}$

Der Krieg in Jugoslawien veränderte die Zusammensetzung der albanischen Bevölkerung in der Schweiz nicht nur durch den Zufluss junger Männer. Viele klassische Fremdarbeiter liessen nun ihre kinderreichen Familien nachkommen, so dass sich die ausgesprochene Männergesellschaft wandelte. Ungünstigerweise fiel dieser grosse zahlenmässige Zuwachs mit der wirtschaftlichen Krise und Arbeitslosigkeit in der Schweiz zusammen.

Für die Schweiz hatte und hat das zur Folge, dass Zehntausende von Kindern aus einer sehr fremdartigen Kultur in den Schulen absorbiert werden müssen, wobei von den Eltern keine Unterstützung erwartet werden kann.

Für die Männer, die z.T. schon seit längerer Zeit alleine hier leben und arbeiten, bedeutet der Familiennachzug zum einen eine Erleichterung und einen emotionalen Gewinn. Sie wissen ihre Familie nun in Sicherheit und haben ihre Frau und die Kinder um sich. Zum andern hat der Familiennachzug für sie aber auch extrem erhöhten Stress zur Folge, und dies aus verschiedenen Gründen:

- Mit demselben tiefen Lohn oder schlimmstenfalls mit dem Arbeitslosengeld oder der Sozialhilfe muss nun eine vielköpfige Familie in der teuren Schweiz unterhalten werden. Der an sich mögliche Familiennachzug wurde aus diesen finanziellen Überlegungen früher gar nicht in Anspruch genommen. Zuwendungen an den Rest der Grossfamilie in der Heimat werden zudem weiterhin erwartet. Der Druck des Clans ist diesbezüglich gross. Als bester Sohn gilt jener, der am meisten Geld schickt. ${ }^{15}$ Diesen Anforderungen nur ungenügend nachkommen zu können, beeinträchtigt das Selbstwertgefühl der Männer und ihr Ansehen bei den anderen.
- Frau und Kinder, die zu Hause traditionell lebten und der Autorität des Grossvaters unterworfen waren, sind nun in einer völlig anderen, aus der Sicht der Albaner verderblichen Umgebung. Konflikte sind deshalb unvermeidlich, v.a. mit den Kindern. Insbesondere die Vater-Tochter-Beziehung ist grössten Spannungen ausgesetzt. Während das Mädchen sich integrieren und zaghaft emanzipieren will, versucht der Vater verzweifelt und nicht selten mit Gewalt, ein Regime aufrechtzuerhalten, wie es in der Heimat üblich ist. Er ist dabei auf sich allein gestellt, ihm fehlt die Grossfamilie, die ihn in seinem Tun unterstützen würde. Und eventuell auch die Erfahrung, denn zu Hause stand er selbst unter der Autorität seines Vaters. Zudem unterscheidet sich seine jetzige Lage dermassen von der Situation in der Heimat, dass für viele Situationen keine Verhaltensmuster vorhanden sind. Tag für Tag muss der Patriarch Entscheidungen treffen, für die er nicht vorbereitet ist, die uns als nichtig erscheinen können, von denen aber seine Ehre abhängt. Private Einladungen und Ausgang kann er verbieten, doch die Schule bringt ihn in die Zwickmühle. Die Tochter sollte schwimmen, an einem OL oder einem Klassenlager teilnehmen. Und was tut man gegen einen Lehrer, der die Tochter gegen den Vater unterstützt?

- Während es früher möglich war, ein weitgehend isoliertes und selbstgenügsames Leben zu führen, von niemand so richtig bemerkt, steigt durch das Familienleben die Kontaktfläche mit der umgebenden Schweizer Gesellschaft enorm. Man muss sich nun mit der Schule auseinandersetzen, allenfalls mit anderen Eltern, mit Hauswarten und Nachbarn, die sich am Treiben der Kinder stören.

- Da fällt es zunächst einmal anscheinend leichter, die Ehefrau unter Kontrolle zu halten, indem sie zu Hause bleiben muss. Doch auf die Dauer kann das nicht gut gehen. In den ländlichen, traditionell lebenden Gegenden, woher die meisten Albaner kommen, wird kein Familienleben nach schweizerischer Art geführt, in welchem die Frau v.a. für das Wohl ihrer Kernfamilie sorgt. Dort leben vielmehr in der Grossfamilie drei Gesellschaftsschichten nebeneinander, nämlich Männer, Frauen und Kinder. Diese drei Gruppen gehen je getrennte Wege, wobei sich die grösseren Kinder um die kleineren kümmern. Nur zu bestimmten Tageszeiten vermischen sich die drei Gruppen. ${ }^{16}$ Die Albanerin in der Fremde ist somit aus ihrer Frauengemeinschaft herausgerissen und muss einen neuen Lebensmittelpunkt finden.

Mit einem Wort: Alle Familienmitglieder sind gezwungen, sich an eine neue Lebenssituation anzupassen und sich somit zu ändern. Gleichzeitig wird

13 Der Bund, 16.1.1999.

14 Der Bund, 16.1.1999.

15 Leuenberger/Maillard, Les damnés, S. 58.

16 Leuenberger/Maillard, Les damnés, S. 30. 
das aber von vielen nicht gewünscht, die das vielfach virtuelle Ziel haben, in die Heimat zurückzukehren. Denn mehr noch als andere Emigranten sind die Albaner mit Kopf und Herz in der Heimat. Sie lebten hier zwanzig und mehr Jahre wie im Wartesaal. Wenn jedoch die Familie da ist, ist das nicht mehr möglich, eine Auseinandersetzung mit der Umgebung wird unumgänglich. Dass die aus einfachen Verhältnissen stammenden, wenig gebildeten und das isolierte Kleinfamilienleben ungewohnten Menschen, ob Männer, Frauen oder Kinder, diese mannigfaltigen Probleme häufig nicht meistern, kann nicht weiter verwundern.

Der Krieg hat die ohnehin beträchtlichen Probleme der albanischen wie auch der anderen jugoslawischen Migranten in grossem Ausmass verschärft. Auch jene Menschen, die schon lange in der Schweiz leben, haben das Geschehen in der Heimat als traumatisierend erlebt, sei es, dass Angehörige oder sie selbst bei einer Heimreise in Mitleidenschaft gezogen wurden, sei es, dass ihr Haus zerstört wurde, für das sie hier gearbeitet hatten, oder weil sie den Zerfall ihrer Heimat beklagen.

Der Imageverlust, der schon vorher eingetreten war, beschleunigte sich durch den Krieg noch mehr. Früher waren die Menschen aus Jugoslawien stolz auf ihre schöne und vielfältige Heimat, die politisch spe- ziell und interessant war, aber nicht zum bösen kommunistischen Osten gehörte und als Ferienland beliebt war. Heute ist einerseits die Heimat erledigt, andererseits das Bild der "Jugos" in der Schweiz auf einem absoluten Tiefpunkt. Dies alles wirkt sich auf das Selbstwertgefühl und somit auch auf die Gesundheit der Menschen negativ aus.

Es kann nicht verwundern, dass diese Immigranten, die in verschiedenen gesellschaftlichen Bereichen Integrationsprobleme haben, im Gesundheitssystem ebenfalls auffallen. Denn, wie die amerikanische Anthropologin Nancy Scheper-Hughes ${ }^{17}$ sagt:

"Krankheit ist kein isoliertes Ereignis, und auch nicht ein unglückliches Scharmützel mit der Natur. Sie ist eine Form der Kommunikation - die Sprache der Organe -, durch welche sich Natur, Gesellschaft und Kultur gleichzeitig äussern. Der individuelle Körper muss sowohl als das unmittelbarste Terrain betrachtet werden, wo soziale Wahrheiten und soziale Widersprüche ausgetragen werden, als auch als der Ort von persönlichem und sozialem Widerstand, Kampf und Kreativität.»

17 Scheper-Hughes N. The Mindful Body. Prolegomena to Future Work in Medical Anthropology. Med Anthropol Q 1987;1:6-41, hier S. 31. 